\title{
Quality control of injection molded eyewear by non-contact deflectometry
}

\author{
A. Speck \\ speck.alexis@gmail.com \\ B. Zelzer
}

\section{A. Langenbucher}

\section{T. Eppig}

occupational eye wear such as safety spectacles are manufactured by injection molding techniques. Testing of the assembled safety spectacle lenses in transmission is state of the art, but there is a lack of surface measurement systems for occupational safety lenses. The purpose of this work was to validate a deflectometric setup for topography measurement, detection of defects and visualization of the polishing quality, e.g. casting indentations or impressions, for the production process of safety spectacles. The setup is based on a customized stereo phase measuring deflectometer (PMD), equipped with 3 cameras with $f^{\prime} 1,2=16 \mathrm{~mm}$ and $\mathrm{f}^{\prime} 3=8.5 \mathrm{~mm}$ and a specified measurement uncertainty of $\pm 3 \mu \mathrm{m}$. Sixteen plastic lenses and 8 corresponding injection molds from 4 parallel cavities were used for validation of the deflectometer. For comparison an interferometric method and a reference standard ( $<\lambda / 10$ super polished) was used. The accuracy and bias with a spherical safety spectacle sample was below $1 \mu \mathrm{m}$, according to DIN ISO 5725-2.2002-12. The repeatability was $2.1 \mu \mathrm{m}$ and $35.7 \mu \mathrm{m}$ for a blind radius fit. In conclusion, the PMD technique is an appropriate tool for characterizing occupational safety spectacle and injections mold surfaces. With the presented setup we were able to quantify the surface quality. This can be useful and may optimize the quality of the end product, in addition to standardized measuring systems in transmission.

[DOI: http://dx.doi.org/10.2971/jeos.2014.14027]

Keywords: PMD, safety spectacle, mold, surface measurement, deflectometry, wave front measurement, surface analysis, injection molding

\section{INTRODUCTION}

Work and activities with possible chemical, thermal or mechanical hazards requires protection with safety spectacles. Occupational eye wear such as safety spectacles are manufactured by injection molding techniques. A typical injection molding machine is equipped with a molding tool which contains a set of parallel injection molds for the polycarbonate spectacles. Despite the high importance of the optical quality of the spectacles, molds are rarely tested for surface quality during the manufacturing process. During the processing of approximately $2 \times 10^{5}$ injection molded parts, quality degradation is mainly being detected by visual and optical inspection in conformity to the european, german or international standards [1]- [4]. Wave-front sensing methods (WF) and optical methods, e.g. optical test bench (OB) may be used for optical inspection of coated and assembled safety devices. The optical bench test is an accredited test used in the spectacle production process. A telescope lens is used for observation of an illuminated test target mounted at a distance of $4.6 \mathrm{~m}$ from the eye protector with an circular measurement field of $20 \mathrm{~mm}$, according to ISO 4854 and EN 167 [4, 5]. The ANSI test is similar using a distance of $10.67 \mathrm{~m}$ [2]. The wave-front system was introduced in a previous publication and was crosschecked and validated to the OB, which is currently used in the production process for quality check of the spectacles. The WF setup is based on a Shack-Hartmann sensor and allows investigation of wave-front aberrations in addition to refractive power data and image quality classification for safety spectacles [6]. However, both optical transmission techniques do not allow a retrospective classification of mold surface quality or injection molding behavior.

Process optimization for a safety spectacle model requires a high amount of iterations until the end product reaches the specified quality. In addition, mold outwear counteracts optimization and degrades quality. Figure 1 shows the deviation plots of re-polished surfaces of a mold for the time period of revision.

In previous publications we proposed a prototype of a deflectometric inspection of mold surfaces $[7,8]$. The deflectometric method allows extraction of height data and detection of defects on the mold surfaces. The standardized optical test bench and the wave-front technique reveal aberrations, for example astigmatism, spherical error or tilt. However, the intended use of this work is to monitor every step in the whole process in order to compare on an equivalent basis. In this study we focused on establishing a deflectometric measurement of specta- 


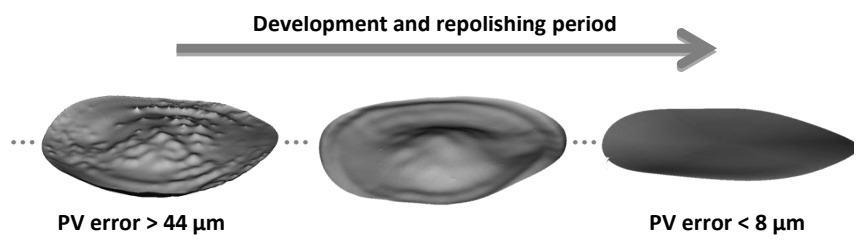

FIG. 1 Deviation plots of a typical preparation and polishing process of safety spectacle molds. Z-axis is magnified by a factor of 400 .

cle topography in the entire process chain. The measurements should be comparable to distinguish surface degradation and the casting impression of the injection molding tool on the basis of the measured lens surface.

The purpose was to validate 3D deflectometry for measuring specular reflecting spectacle surfaces for the detection of defects and surface outwear with the aim of optimizing the spectacle development and production.

\subsection{Measurement technique}

The setup is based on the principle of the phase measuring deflectometry (PMD). A screen with a fringe pattern (sinusoidal profile) is reflected by the object under test and the reflex image is captured by a camera (Figure 2). Local slope data can be calculated from the observed surface reflections (distortion of the sinusoidal pattern). Further, curvature $c$ and especially surface (mean) power $P_{S}$ (Eq. 1) can be calculated through the derivative of the slope data (Eq. 2) $[9,10]$.

$$
\overline{P_{S}}=\frac{(n-1) \times\left(c_{1}+c_{2}\right)}{2} ; P_{s}=\frac{n-n^{\prime}}{R}
$$

with refractive indices $n, n^{\prime}$

curvature $\frac{1}{R} ;\left(\right.$ principal curvatures $\left.c_{1}, c_{2}\right)$

$$
\overrightarrow{x_{u}}=\left(\frac{d x}{d u}, \frac{d y}{d u}, \frac{d z}{d u}\right)
$$

with first derivative $\overrightarrow{x_{u}}$ of a surface $\vec{x}$

In this example a surface in world coordinates $(x, y, z)$ was used, which is parameterized by $(u, v)$. From such a surface, the principal curvatures (Eq. 3) are calculated with the Weingarten mapping [11].

$$
\begin{aligned}
& \text { priniple curvatures } c 1, c 2=H \pm \sqrt{H^{2}-K} \\
& \text { with Gaussian curvature } K=\operatorname{det}(A) \\
& \text { Mean curvature } H=\frac{1}{2} \operatorname{tr}(A)
\end{aligned}
$$

The local height data is then calculated with an integral of the slope data respectively [9]-[12].

\section{MATERIAL AND METHODS}

A commercial available PMD sensor was the basis of the measurement system (SpecGAGE3D, 3D-Shape GmbH, Erlangen Germany). The customized setup is based on an industrial black and white screen as pattern generator and three cameras $\left(f_{1,2}=16 \mathrm{~mm}, f_{3}=8.5 \mathrm{~mm}\right.$ ) for distortion detection (Figure 3 ). A mean repetition accuracy of $1.6 \times 10^{-3} \mathrm{~m}^{-1}$ is specified for

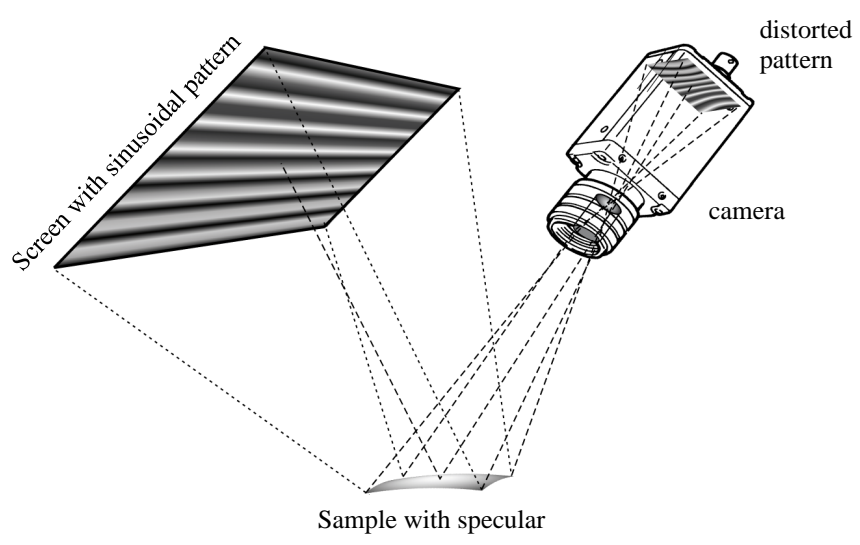

FIC. 2 Simplified illustration of the PMD technique. The industrial flat screen generates a sinusoidal pattern. The distorted pattern is observed by the camera. Modified from Seßner [14].
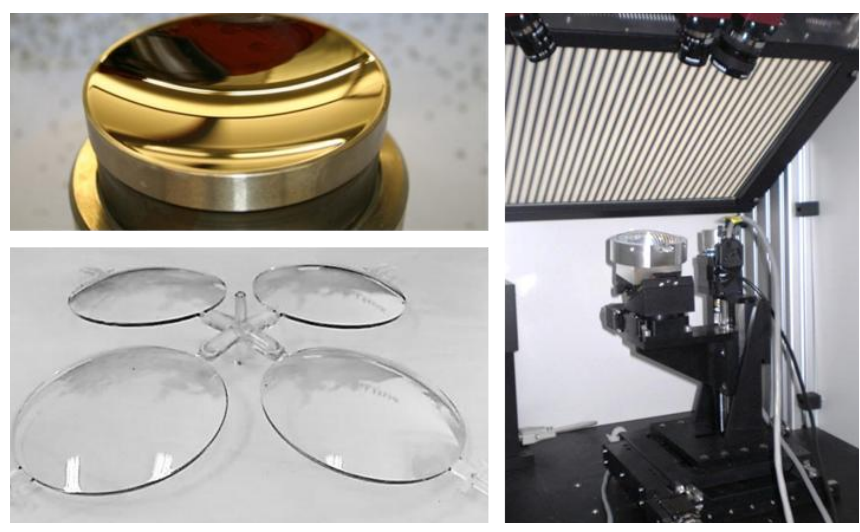

FIC. 3 Left side: Concave side of the injection molding tool and four cavities of the injected safety spectacles from the entire tool. Right side: Test setup of the phase measuring stereo deflectometry including the industrial $b / w$ screen for sinusoidal pattern generation, three cameras for observing the distorted pattern, the five axis positioning system for defined mold and spectacle movement between the surface measurements and the holder for the spectacle samples.

the sensor when measuring within a working range of concave radius $r_{\text {ccomax }}$ from $-\infty$ to $-40 \mathrm{~mm}$ and convex radius $r_{\text {cvxmax }}$ from $40 \mathrm{~mm}$ to $\infty$, for high specular reflecting surfaces without multilayer surfaces. The measurement uncertainty is specified with $\pm 3 \mu \mathrm{m}$ for a lateral measurement range of $80 \mathrm{~mm} \times 80 \mathrm{~mm}$ [13]. For specimen positioning, we used a customized developed five axis stage (micron positioning), referenced on the sensor axis $(x, y)$ and defined adapter for repositioning the samples in a micrometer range (Figure 3 ). SoftPMD (Max Planck Research Group, Friedrich-AlexanderUniversity of Erlangen-Nuremberg, Germany) Minitab ${ }^{\circledR}$ (Version 16.2.3) and Matlab (R2011a) were used for graphical and statistical analysis. To test the calibration of the setup and to quantify the accuracy, we used a commercial lens S1LPX0258 (Sill Optics GmbH \& Co. KG) as a reference standard $(<\lambda / 10$ super polished, modified backside), measured by interferometric methods.

\subsection{Samples and measuring process}

Sixteen plastic lenses (32 optical surfaces) and the corresponding 8 injection molds were measured in this study. These samples were derived from a single optical design and produced 


\begin{tabular}{|cccccccccccc|}
\hline \multicolumn{2}{|c}{ definition } & unit & V1/V7 & V2/V8 & V3/V9 & V4/V10 & V5/V11 & V6/V12 & mean & SD \\
\hline cam2 & PV error & {$[\mu \mathrm{m}]$} & 4.710 & 4.714 & 4.679 & 4.706 & 6.667 & 4.686 & 5.027 & 0.803 \\
& radius & {$[\mathrm{mm}]$} & 67.603 & 67.603 & 67.603 & 67.603 & 67.601 & 67.603 & 67.603 & $8.17 \cdot 10^{-4}$ \\
\multirow{2}{*}{ cam3 } & PV error & {$[\mu \mathrm{m}]$} & 4.376 & 4.393 & 6.677 & 4.318 & 6.696 & 4.378 & 5.140 & 1.20 \\
& radius & {$[\mathrm{mm}]$} & 67.604 & 67.604 & 67.601 & 67.604 & 67.601 & 67.604 & 67.603 & $15.5 \cdot 10^{-4}$ \\
\hline
\end{tabular}

TABLE 1 Accuracy check with the reference standard for camera two and camera three.

in 4 parallel cavities (Figure 3). Molds with concave radii of $r_{c c v}=88.03 \mathrm{~mm}$ and convex radii of $r_{c v x}=87.67 \mathrm{~mm}$ were measured (Material: M390, 60 hrc). The cavity groups were polished by different techniques. Cavities two to four were taken directly from the production process with various lifetimes and shot volumes. They were rotationally polished with a round polishing mask and a manual finishing through an experienced polisher. Cavity one was polished with a fully automated polishing technique (robot with polishing head) $[15,16]$. The polishing path was arranged in a circular manner. Especially for finishing, a manual revision was performed. The mold and plastic samples were aligned in a defined holder in a constant position and the measurements were acquired consecutively using identical parameters. To avoid backside reflections from the polycarbonate transparent spectacles, we used an index matching gel (coupling gel) and black felt as background.

\subsection{Analysis}

\subsubsection{Accuracy}

The specified accuracy was checked directly after the calibration with a laser grade reference standard (sample: S1LPX0258 from Sill Optics $\mathrm{GmbH} \& \mathrm{Co}$. KG) with a convex radius of $67.608 \mathrm{~mm}$ and a super polished surface of better than $\lambda / 10$. The backside was unpolished and painted black to avoid reflections. The measurement uncertainty was calculated for camera 2 (cam2) and camera 3 (cam3) separately with a series of 12 measurements. The samples were aligned in a constant position and the measurements were acquired consecutively using identical parameters. We analyzed the accuracy through the systematic error of measurement (bias of the measuring cameras). The measurements were done parallel with all three cameras. Stereo-deflectometry requires at least a second camera (cam1) to determine the absolute position in space, also referred to as absolute phase measuring deflectometry $[11,17]$. Therefore each camera picture (from cam 2 and cam3) in combination with the camera for absolute position measurement (cam1) was used to calculate the slope data. For measurement error estimation mean values and mean differences (peak to vally error) were calculated with a comparison of the measurements and the target radius of the reference standard. A gauge bias study was performed according to DIN ISO 5725:2002 in order to compare the accuracy of camera 2 and 3 [18].

In a last step a spherical convex sample of the injected parts was used. Two measurement series (V1-V5, V6-V10) were analyzed by subtracting the best-fit sphere. For repeat accuracy (first series) the sample was aligned in a constant position and for repeatability (second series) in arbitrary position.

\subsubsection{Sample Analysis}

The main part of the analysis comprises the measurement of the safety spectacle samples and their corresponding molds. The 8 molds associated to cavities 1 to 4 were measured as well as the corresponding convex and concave sides of the spectacles from the 4 cavities. A scatterplot was drawn from the wave-front RMS data (wave-front system) and the surface RMS data deflectometric system) for extraction of the polishing quality and difference between concave and convex sides of the molds and the samples respectively. The second part of the sample analysis contains the statistical evaluation of cavity one and three in terms of the residuals (mean error) and the RMS. Pseudo color plots of the residuals, including peak to vally value (PV) and local deviations are presented. Curvature analysis of the measured mold and spectacle surfaces (cavity one and three) revealed molding and forming indentations. An additional structure comparison to the mold surface exposed casting indentations.

\section{RESULTS}

\subsection{Measurement uncertainty}

The interferometric measurement (null lens: JENfizar 6 " f/0.75, Jenoptik, Jena, Germany) of a reference lens $(\mathrm{r} 1=67.608 \mathrm{~mm})$ element resulted in a PV deviation of about $0.05 \mu \mathrm{m}(0.08 \lambda)$ and $0.006 \mu \mathrm{m}$ RMS $(0.01 \lambda)$ (Figure 4(a)). Table 1 represents the deflectometer accuracy check. Camera two resulted in a measurement uncertainty of $5.0 \mu \mathrm{m}$ PV and camera three $5.1 \mu \mathrm{m}$ PV. The measured mean radius was about $67.603 \mathrm{~mm}$ for both cameras (Figure 4(b)).

The results of the repeatability and accuracy check with a spherical safety spectacle sample are shown in Table 2. The difference between the best-fit radius and the measurement was provided in terms of PV error and the measured radius. Statistics are provided by mean, the absolute deviation from the average, standard deviation (SD) and median values. The measurement uncertainty was below $1 \mu \mathrm{m}$ for the samples (abs. PV error $800 \mathrm{~nm}$ ). The second series, with arbitrary positioning between the measurements, showed an error of about $2.1 \mu \mathrm{m}$. The repeatability of the fitted radius was $35.7 \mu \mathrm{m}$.

\subsection{Safety spectacle samples}

The scatter plot of all four cavities showed a high difference in the surface quality between the two different polishing techniques (Figure 5). The surface measurement with the PMD method showed an RMS error up to $12.5 \mu \mathrm{m}$ for cavity one (concave side). In comparison, the mold of the other cavities 

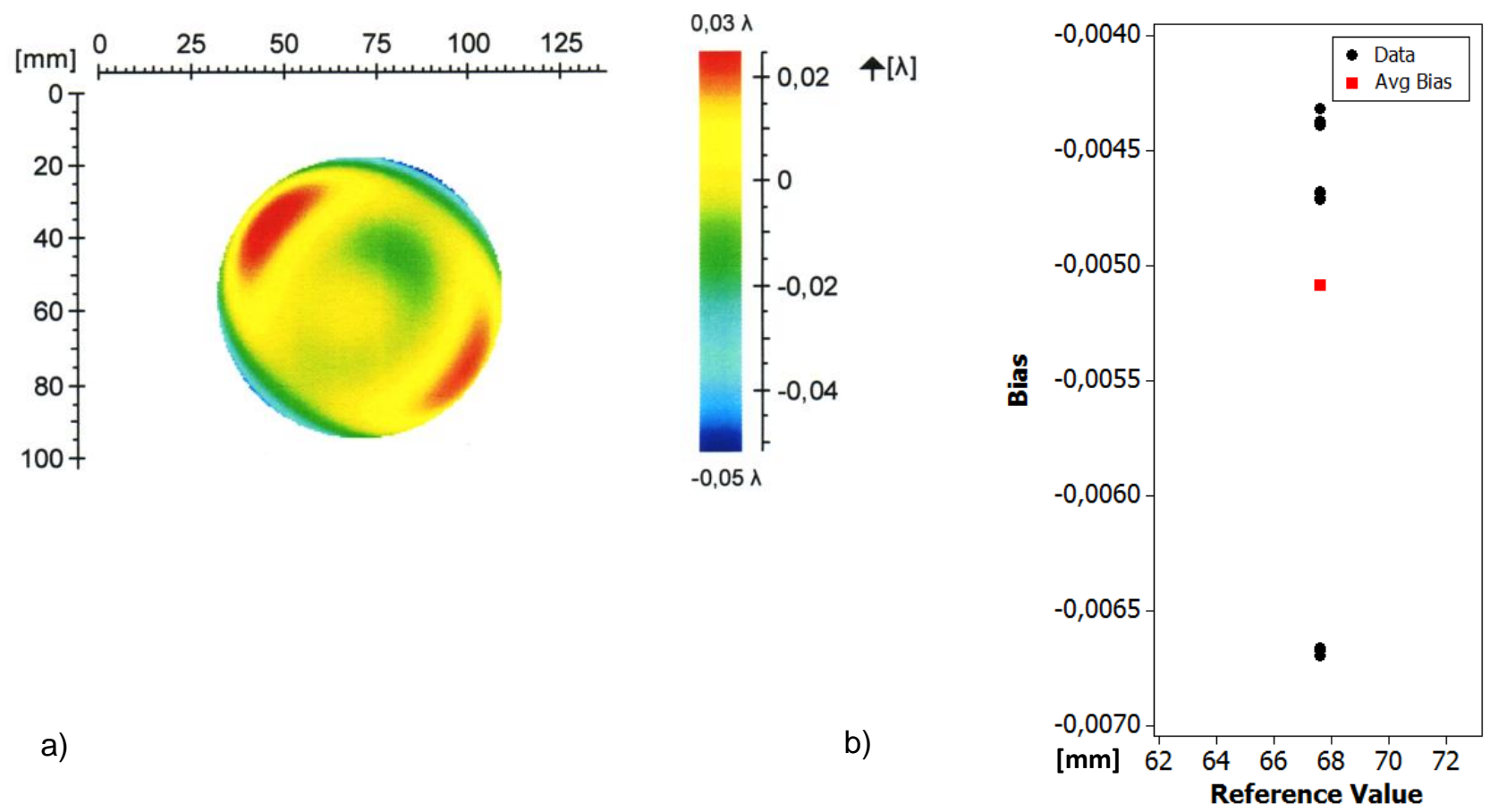

FIG. 4 a) Color coded deviation plot of the interferometric measurement of a reference standard (Sill 0ptics $\mathrm{GmbH}$ a Co. $\mathrm{KG} ; \lambda=632.8 \mathrm{~nm}$ ) with a convex radius of $67.608 \mathrm{~mm}$. b) Individual value plot of the Bias calculation for the PMD sensor with the reference standard.

\begin{tabular}{|c|c|c|c|c|c|c|c|c|c|c|c|}
\hline \multirow[t]{2}{*}{ definition } & \multicolumn{3}{|c|}{ unit } & \multicolumn{3}{|c|}{ first series } & \multirow[b]{2}{*}{ V5 } & \multicolumn{4}{|c|}{ accuracy statistics } \\
\hline & $\#$ & & V1 & V2 & V3 & V4 & & mean & abs. PV & $\mathrm{SD}$ & median \\
\hline \multirow[t]{3}{*}{ parameter } & radius & {$[\mathrm{mm}]$} & 88.681 & 88.661 & 88.675 & 88.676 & 88.674 & 88.673 & 0.005 & 0.007 & 88.675 \\
\hline & SD & {$[\mathrm{mm}]$} & 0.016 & 0.017 & 0.017 & 0.017 & 0.017 & 0.017 & 0.000 & 0.000 & 0.017 \\
\hline & PV & {$[\mathrm{mm}]$} & 0.074 & 0.075 & 0.077 & 0.077 & 0.076 & 0.076 & 0.001 & 0.001 & 0.076 \\
\hline \multirow[t]{4}{*}{ error } & mean & [mm] & 0.005 & 0.005 & 0.005 & 0.005 & 0.005 & 0.005 & 0.000 & 0.000 & 0.005 \\
\hline & SD & {$[\mathrm{mm}]$} & 0.016 & 0.016 & 0.016 & 0.016 & 0.016 & 0.016 & 0.000 & 0.000 & 0.016 \\
\hline & & unit & \multicolumn{5}{|c|}{ first series } & \multicolumn{4}{|c|}{ accuracy statistics } \\
\hline & $\#$ & & V6 & V7 & V8 & V9 & V10 & mean & abs. PV & $\mathrm{SD}$ & median \\
\hline \multirow[t]{3}{*}{ parameter } & radius & [mm] & 88.786 & 88.868 & 88.892 & 88.864 & 88.850 & 88.852 & 0.027 & 0.036 & 88.864 \\
\hline & SD & {$[\mathrm{mm}]$} & 0.018 & 0.019 & 0.019 & 0.019 & 0.019 & 0.019 & 0.001 & 0.001 & 0.019 \\
\hline & PV & {$[\mathrm{mm}]$} & 0.079 & 0.084 & 0.088 & 0.086 & 0.084 & 0.084 & 0.002 & 0.003 & 0.084 \\
\hline \multirow[t]{2}{*}{ error } & mean & {$[\mathrm{mm}]$} & 0.005 & 0.006 & 0.006 & 0.006 & 0.006 & 0.006 & 0.000 & 0.000 & 0.006 \\
\hline & SD & {$[\mathrm{mm}]$} & 0.017 & 0.018 & 0.018 & 0.018 & 0.018 & 0.018 & 0.000 & 0.001 & 0.018 \\
\hline
\end{tabular}

TABLE 2 Best-fit repeatability check with a spherical safety spectacle sample.

were better except one outlier of cavity four (RMS error of about $18 \mu \mathrm{m})$. Cavity two and tree had almost the same RMS error up to $10 \mu \mathrm{m}$. Cavity four had a higher statistical spread between $6 \mu \mathrm{m}$ and $18 \mu \mathrm{m}$. Table 3 shows the comparison of the descriptive statistics for cavity one and three. The wavefront measurement showed a high difference between cavity four and one of about $1.2 \mu \mathrm{m}$ RMS. In Figure 6 a comparison of the spectacles residuals of the mean curvatures is shown for cavity one and three, in each case one example from the batch (cavity two and four were similar to cavity three, due to the same polishing technique). In general, cavities two to four had no local form errors. Spectacle lens surfaces from cavity one had different types of structures. Figure 7 shows the comparison of the molds from cavity one and three.

The local structures were similar to the structures on the polycarbonate spectacles. Figure 8 shows the structures and patterns on the mold and spectacle residuals exemplary for one side of cavity one of the injection molding tool (scaled z-axis, factor 500). Note the forming on the safety spectacle model. Only one type of structure was marked in every part of the figure, for reasons of clarity. The inspection of the casting impression and the surface shows especially four types of structures:

- one horizontal stripe (with a deflection) on both sides

- a couple of vertical stripes on the entire surface on both sides

- pickles on the concave spectacle respective convex mold surface

- a sombrero-like figure in the middle of both sides (one side is inverted)

- circular paths / rings on the entire surface on both sides 


\begin{tabular}{|c|c|c|c|c|c|c|}
\hline definition & surface & cavity \# & unit & mean & SD & median \\
\hline \multirow{2}{*}{ residuals } & \multirow{2}{*}{ concave } & 1 & {$[\mu \mathrm{m}]$} & -2.88 & 0.73 & -2.77 \\
\cline { 3 - 7 } & & 3 & {$[\mu \mathrm{m}]$} & -1.05 & 1.23 & -1.04 \\
\hline \multirow{2}{*}{ residuals } & \multirow{2}{*}{ convex } & 1 & {$[\mu \mathrm{m}]$} & 2.42 & 0.18 & 2.46 \\
\cline { 3 - 7 } & & 3 & {$[\mu \mathrm{m}]$} & -0.54 & 0.22 & -0.55 \\
\hline \multirow{2}{*}{ RMS } & \multirow{2}{*}{ concave } & 1 & {$[\mu \mathrm{m}]$} & 12.54 & 1.07 & 12.33 \\
\cline { 3 - 7 } & & 3 & {$[\mu \mathrm{m}]$} & 6.11 & 1.81 & 6.06 \\
\hline \multirow{2}{*}{ RMS } & \multirow{2}{*}{ convex } & 1 & {$[\mu \mathrm{m}]$} & 12.23 & 2.23 & 12.14 \\
\cline { 3 - 7 } & & 3 & {$[\mu \mathrm{m}]$} & 2.62 & 0.52 & 2.77 \\
\hline
\end{tabular}

TABLE 3 Descriptive statistics for spectacles of cavity one and cavity three for the PMD measurement.

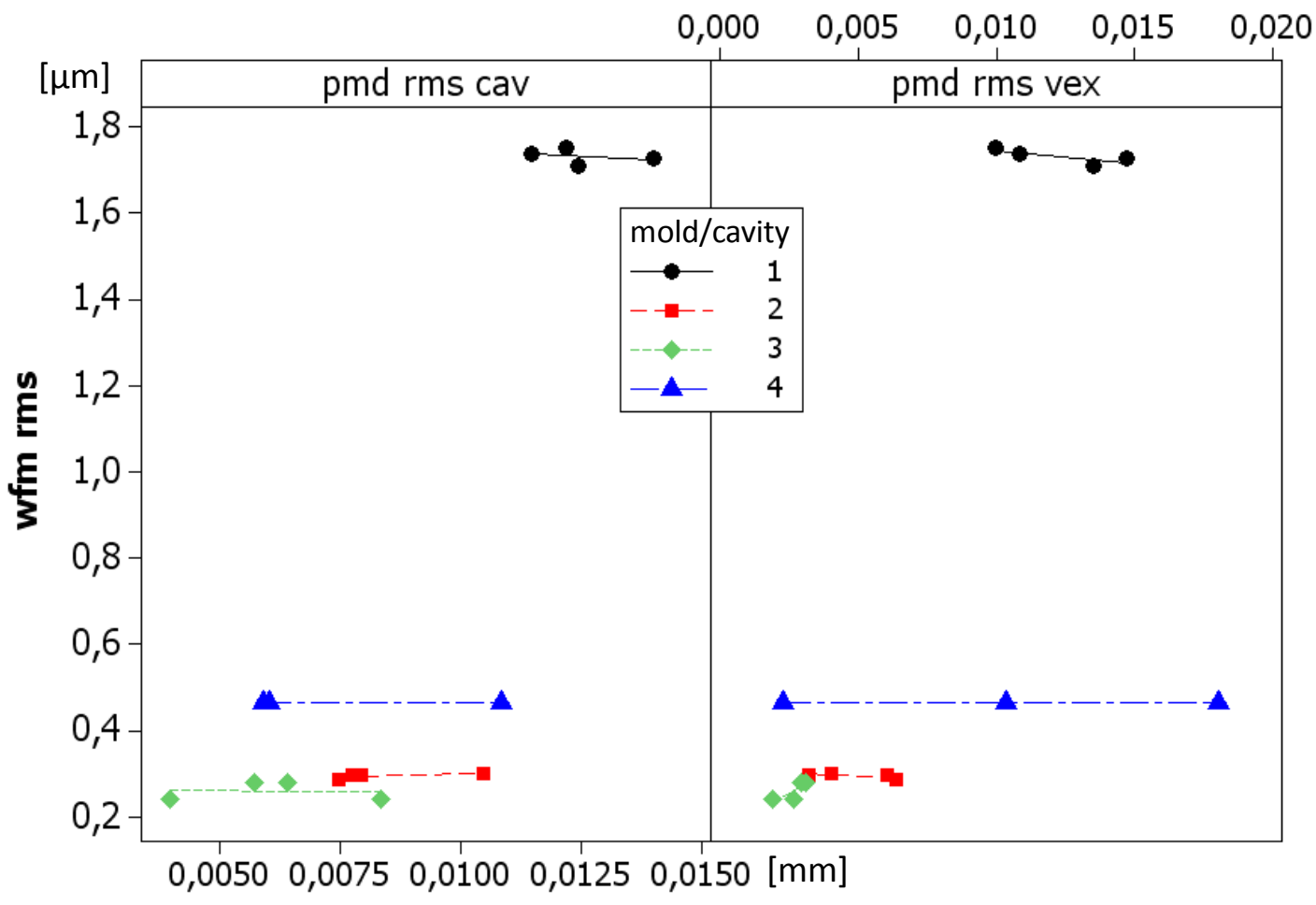

FIG. 5 Scatter plot of the wave-front measurement (wfm rms) versus topography measurement (pmd rms) for both sides of the plastic lenses (concave=cav, convex=vex).

\section{DISCUSSION}

We applied the phase measuring deflectometry for measuring safety spectacle glasses in the production process. The measured polycarbonate topographies disclosed surface degradations, casting impressions of the injection molding tool and we were able to distinguish different abrasive effects between the cavities in one mold. To our knowledge, there are no published results for measuring safety spectacle lenses by stereo phase measuring deflectometric methods.

A five axis stage was calibrated on the PMD sensor axis for sample positioning. The samples could be repositioned with about $1 \mu \mathrm{m}$ precision. The calibration test of the setup was performed with a reference standard (modified backside, $<\lambda / 10$ ). Sixteen plastic lenses were measured in this study and 8 injection molds of the safety spectacles. To quantify the accuracy, the measured and analyzed surface data of the reference standard was compared to an interferometric measurement which outperformed the PMD sensor in terms of absolute accuracy. For validation and investigation of conditions between the cavities of the tool, we additionally compared the results to a previously published method in transmission [6]. Cavity one was produced and polished with a fully automated polishing technique. Cavity two to four were taken from the production process with various lifetimes and shot volumes, produced with a manual manner.

The accuracy check with a reference element $(R=67.608 \mathrm{~mm})$ resulted in a peak-to-valley deviation of about $0.05 \mu \mathrm{m}$ and $0.006 \mu \mathrm{m}$ RMS. Camera two of the PMD setup resulted in a measurement uncertainty of $5.0 \mu \mathrm{m}$ PV and camera three $5.1 \mu \mathrm{m}$ PV. The measured mean radius was about $67.603 \mathrm{~mm}$ for both cameras. The measurement uncertainty, with a spherical safety spectacle sample, was below $1 \mu \mathrm{m}$. The second series, with arbitrary positioning between the measurements, showed an error of about $2.1 \mu \mathrm{m}$. The repeatability of the fitted radius was $35.7 \mu \mathrm{m}$.

The main part of the analysis comprised the measurement of the safety spectacle samples and their mold cavities. A scatterplot was drawn from the wave-front RMS data (wave-front system) and the surface RMS data (deflectometric system) for extraction the polishing quality and difference between con- 

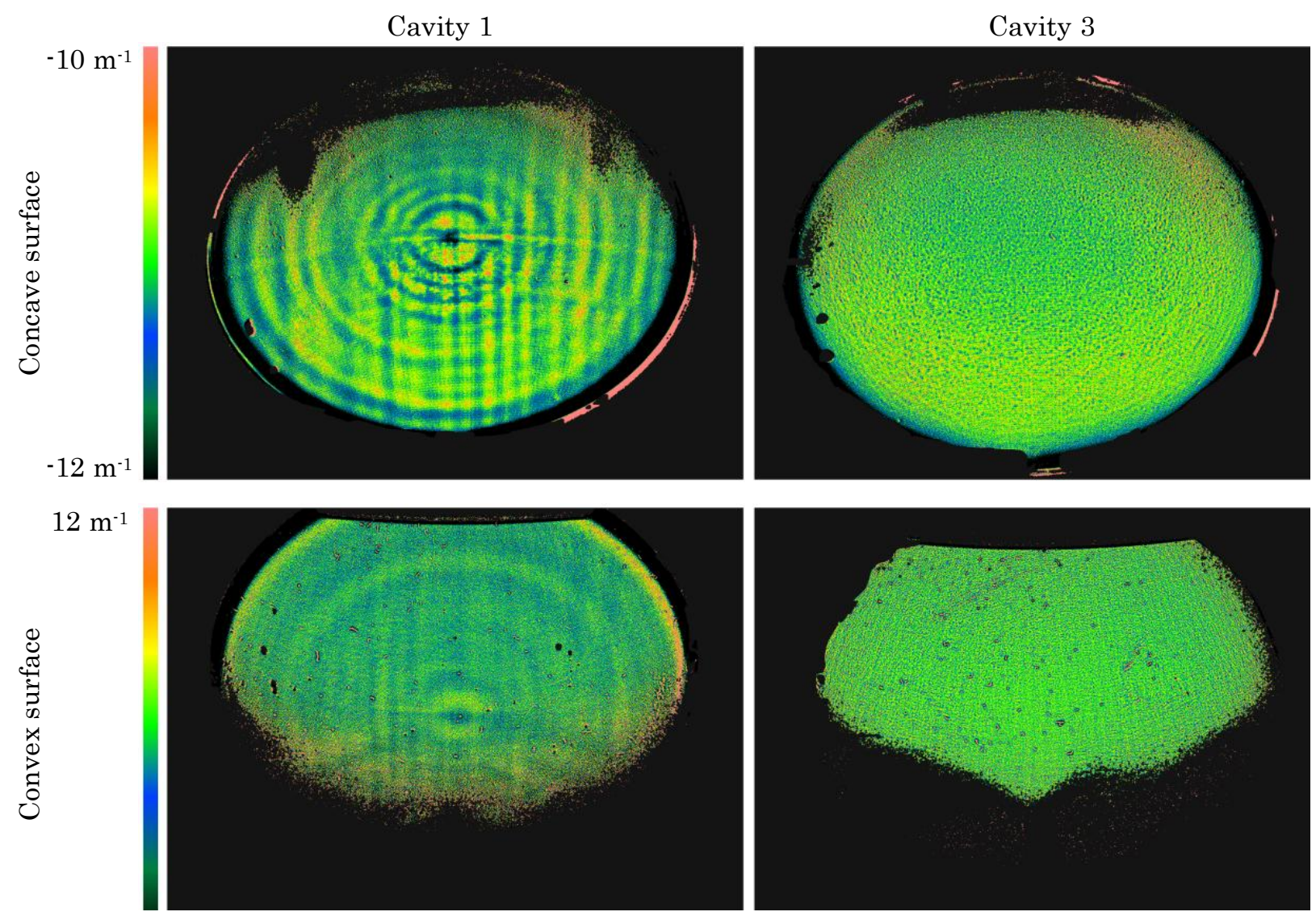

FIG. 6 Mean curvature analysis of the spectacle front- and back side for cavities one and three (one example of each batch). Local form characteristics of cavity one are obvious. Cavity two and four were similar to cavity three, due to the same polishing technique.

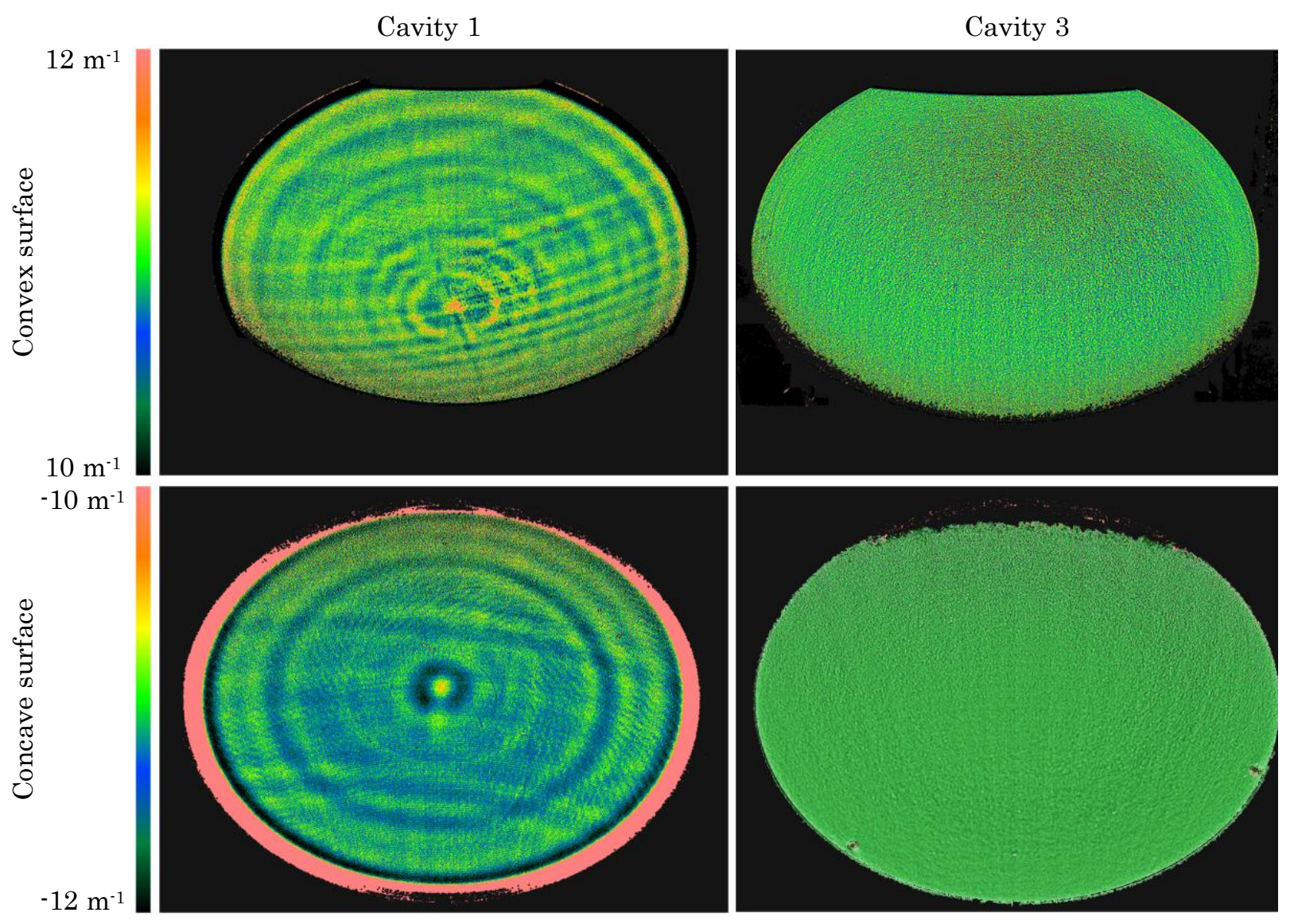

FIG. 7 Mean curvature analysis of the concave and convex molds for cavities one and three. The local form characteristics of cavity one are obvious. Cavity two and four were similar to cavity three, due to the same polishing technique. 


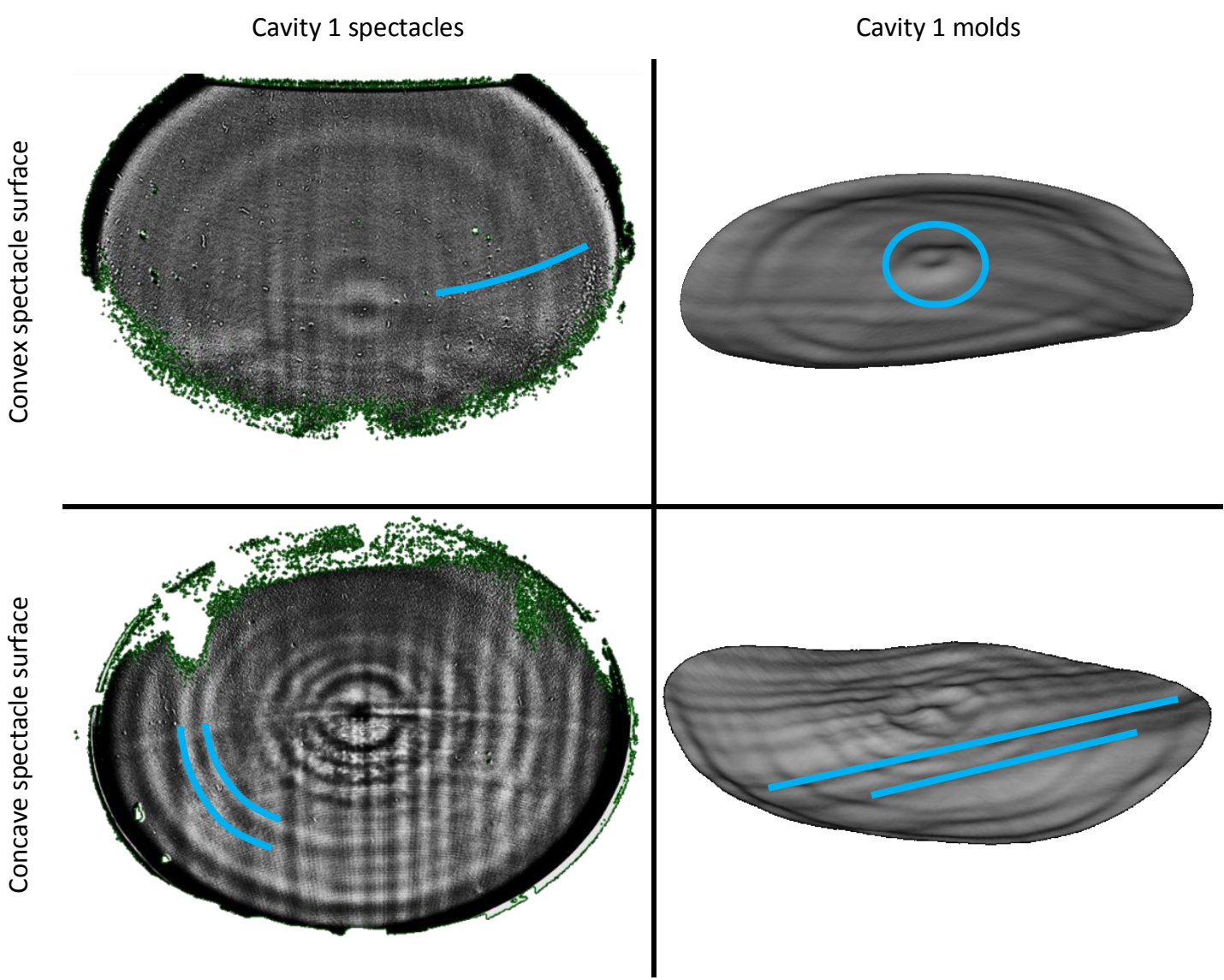

FIG. 8 Structure and injection forming comparison of cavity one. Left: curvature analysis of the spectacle surfaces. Right: height data respective residuals of the mold surface (subtracted target sphere, scaled z-axis times 500).Mean curvature analysis of the spectacle front- and back side for cavity one and cavity three (one example of each batch). The local form characteristics of cavity one can be seen. Cavity two and four were similar to cavity three, due to the same polishing technique.

cave and convex sides of the molds respective the samples. This resulted in a high difference in the surface quality, especially between the two different polishing techniques. Cavity one showed an RMS error of up to $12.5 \mu \mathrm{m}$ and cavity four had a statistical spread between $6 \mu \mathrm{m}$ and $18 \mu \mathrm{m}$. This might be the result of an outlier value as PMD measurements have a higher variability, due to the measurement technique and the noise.

We compared the results from the PMD-sensor with a previously published wave-front system in order to find a correlation between topography and transmission measurement [6]. We estimated differences between the four cavities and their polishing techniques. We expected a difference, due to the more difficult polishing process for convex forms. The polishing agent containing the abrasive particles (crystal, etc.) is running off the convex surface whereas a pooling can be observed with concave forms. Surprisingly, concave convex molds showed a nearly equal polishing quality in respect to the wave-front measurement and the PMD measurement. The comparison of the descriptive statistics for cavity one and three resulted in almost no difference between the concave and convex sides of the spectacle lenses.

However, due to the minor front surface reflection of the spectacle material, the maximum measurement angles and diameters of the samples were limited. The specular reflection of the metal surface of the molds provides better signal to noise ratio and therefore larger measurement angles and sample diameters.

Curvature plots emphasized the local (mean) curvatures and structures of the measured surfaces. Especially high differences such as scratches, structured defects or blemishes were visible with this method on cavity one molds and spectacles. All in all, the statistical results were confirmed due to a high local irregularity. We were able to identify 4 representative defect structures such as horizontal stripes on both sides, vertical stripes on the entire surface, pickles, sombrero-like figures in the middle part and circular paths and rings. We believe most of the structures were caused by the turning and milling process before the polishing. We suppose the material removal was not sufficient, especially in the middle (sombrero figure), therefore the automated polishing technique requires further optimization.

\subsection{Limitations}

Our study had some limitations. Incomplete index matching might cause calculation errors. A software algorithm for thicker samples $(>2 \mathrm{~mm})$ to distinguish the second reflected pattern for safety spectacles with a thickness of $1.5 \mathrm{~mm}$ to $2.9 \mathrm{~mm}$ was available. However, drawbacks of this algorithm are residual errors and a reduction of accuracy. We do not now the direct effect and the principle has to be proofed for safety spectacle concave/convex radii between $58 \mathrm{~mm}$ and ap- 
prox. $200 \mathrm{~mm}$, before applying this algorithm instead of index matching or coupling to reduce the back side reflex.

Another limitation were various coatings of the spectacle sides, depending on the characteristics and properties of the safety spectacle. In the introduction we mentioned the entire monitoring of the injection process chain. This also includes the measurement of the specialized and coated safety spectacles, for example anti-reflex or anti-fog coatings. With state of the art deflectometric methods, it is not possible to prevent multiple reflexes from the different layers. On the other hand, anti-reflex coating decrease the surface reflections and therefore affect measurement accuracy.

However, we demonstrated that the PMD setup is sufficient for measuring occupational safety spectacle lenses. We believe that measurement results are helpful to distinguish metal surfaces and the casting impression of the injection molding tool. Further work will address long term studies with molds respective injection molded parts. We plan to test tinted and coated samples and to compare the results with reference measurement systems. The index matching process still needs to be optimized, with the objective of an automated gel dispenser, automated positioning and measurement. This includes investigations to stitching - algorithms for larger lateral measurement areas.

\section{CONCLUSION}

The presented setup was able to detect surface structures and to differentiate polishing techniques, worn out molds and their casted spectacles. To quantify the quality of the surfaces measurements of both molds and spectacle lenses could be useful and may be representative for the quality of the end product, in addition to measurements of the assembled spectacle in transmission. However, wave front and optical bench tests cannot be replaced.

In conclusion, the PMD technique is an appropriate tool for measuring and characterizing occupational safety spectacle and injections mold surfaces.

\section{ACKNOWLEDGMENTS}

This study was financially supported by a grant of the Bavarian Research Foundation (AZ-874-09), Munich, Germany. The authors wish to thank Uwe Friedlein, Helmut Illauer, Bernd Hutzler, Günther Wolkersdorfer, Stefanie Daniel and Georg Haubner (Uvex Arbeitsschutz $\mathrm{GmbH}$ ) for supporting and providing the injection molding tools and spectacles. Special thanks to Jens Götzelmann and Dr. Stefan Schäfer (Uvex Arbeitsschutz $\mathrm{GmbH}$ ) for scientific discussion and to Dr. Annette Walter and Sill Optics GmbH \& Co. KG for providing interferometric data of the calibration lens.
[2] American National Standard, "Occupational and educational personal eye and face protection devices - spectacles," ANSI Z 87.1 (2003).

[3] Deutsches Institut für Normung e.V., "Personal eye protection specifications," DIN EN 166 (2001).

[4] Deutsches Institut für Normung e.V., "Personal eye protection optical test methods," DIN EN 167 (2001).

[5] International Standard, "Personal eye-protectors - Optical test methods," ISO 4854-1981 (1981).

[6] T. Eppig, K. Zoric, A. Speck, B. Zelzer, J. Götzelmann, D. Nagengast and A. Langenbucher, "Wave-front analysis of personal eye protection," Opt. Express 20, 17806-17815 (2012).

[7] A. Speck, B. Zelzer, M. Speich, R. Börret, A. Langenbucher, and T. Eppig, "Deflectometric analysis of high volume injection molds for production of occupational eye wear," Z. Med. Phys. 23, 314323 (2013).

[8] B. Zelzer, A. Speck, A. Langenbucher, and T. Eppig, "Theoretical model for design and analysis of protectional eyewear," Z. Med. Phys. 23, 120-128 (2013).

[9] J. Kaminski, M. Knauer, and G. Häusler, "Calculating curvatures from discrete slope data," in Lehrstuhl für Optik - Annual Report 2003, M. Eberler, and G. Gardavsky, eds., 31 (Chair of Optics, University of Erlangen-Nuremberg, 2003).

[10] M. Knauer, and J. Kaminski, "Measurement of eye glasses with phase measuring deflectometry," in Lehrstuhl für Optik - Annual Report 2003, M. Eberler, and G. Gardavsky, eds., 30 (Chair of Optics, University of Erlangen-Nuremberg, 2003).

[11] M. Knauer, J. Kaminski and G. Häusler, "Absolute phase measuring deflectometry," DGa0 Proceedings 105, A15 (2004).

[12] C. Horneber, M. Knauer, and G. Häusler, "Phase Measuring Deflectometry: a new method to measure reflecting surfaces," Proc. SPIE 5457, 366-376 (2004).

[13] R. Seßner, "Direction coded Deflectometry by telecentricity," (doctoral thesis, Friedrich-Alexander-University Erlangen-Nuremberg, 2009).

[14] M. Knauer, and K. Veit, "Vermessung spiegelnder Oberflächen eine Aufgabe der optischen 3D-Sensorik," Photonik 4, 62-64 (2004).

[15] M. Speich, and R. Börret, "Mould fabrication for polymer optics," J. Europ. Opt. Soc. Rap. Public. 6, 11050 (2011).

[16] M. Speich, R. Börret, A. K. M. Desilva, D. K. Harrison, and W. Rimkus, "Precision Mold Manufacturing for Polymer Optics," Mater. Manuf. Process. 28, 529-533 (2013).

[17] A. Speck, B. Zelzer, M. Kannengießer, A. Langenbucher, and T. Eppig, "Inspection of freeform intraocular lens topography by phase measuring deflectometric methods," Appl. 0pt. 52, 42794286 (2013).

[18] Deutsches Institut für Normung e.V., "EN-Accuracy (trueness and precision) of measurement methods and results - Part 2: Basic method for the determination of repeatability and reproducibility of a standard measurement method," DIN ISO 5725-2.2002-12 (2002).

\section{References}

[1] International Standard, "Personal eye-protectors - Specifications," ISO 4849-1981 (1981). 